\title{
On the possibility of the existence of the spatially periodical Bose condensate
}

\author{
A.S.Peletminsky ${ }^{1}$, S.V.Peletminsky ${ }^{2}{ }^{*}$, Yu. V.Slyusarenko ${ }^{2 \dagger}$ \\ 1 Electrophysical Scientific and Technical Centre \\ of the National Academy of Sciences of Ukraine, \\ 61002 Kharkiv, Ukraine \\ 2 National Science Centre, "Kharkiv Institute of Physics and Technology", \\ 61108 Kharkiv, Ukraine
}

Received March 6, 2000

The possibility of constructing the theory relating to a spatially periodical Bose condensate in the model of a weakly nonideal Bose gas is studied. Equations for determining a spatially periodical order parameter at the temperature $T=0$ are formulated and their one-periodical solutions are obtained based on the isolated Bose condensate method. Here it is possible to neglect the contribution of quasiparticles to the thermodynamics of a boson system. The problems of thermodynamic stability of the spatially periodical Bose condensate are presented in short.

Key words: weakly nonideal Bose-gas, isolated Bose-condensate method, spatially periodic Bose-condensate, thermodynamic stability

PACS: 03.75.Fi, 05.30.Jp, 05.70.a

\section{Introduction}

Recently the experiments have been carried out where the existence of the BoseEinstein condensation phenomena in an ideal gas at temperature near $2 \cdot 10^{-8} \mathrm{~K}$ was proved [1]. This fact makes it interesting to turn again to the Bose-Einstein condensation problem in the model of a weakly nonideal Bose gas. Within this model the microscopic theory of superfluidity was constructed by N.N.Bogolyubov with the use of a quasiaverage method. Applying the microscopic approach which contains the quantum Gibbs distribution as a fundamental aspect it was shown [4] how the basic equations of motion for a quantum crystal can be obtained. The phenomenological theory of the quantum crystal was described in [5].

*E-mail: spelet@kipt.kharkov.ua

${ }^{\dagger}$ E-mail: slusarenko@kipt.kharkov.ua 
In the present work we want to touch upon the problem of constructing the thermodynamics of the one-periodical quantum crystal in the model of a weakly nonideal Bose gas. Here we consider the questions connected with the description of spatially periodical structures at $T=0$ which correspond to the existence of the spatially periodical Bose condensate. The problems of thermodynamic stability of the states studied are also briefly considered.

It would be interesting to construct the hydrodynamics equations for the oneperiodical solutions, to generalize the theory for the case of two and three-periodical states, as well as to clarify the effect of the external magnetic field on these states. The latter task was considered for the spatially homogeneous Bose condensate in [6].

We also want to emphasize that spatially periodical states in the fermion systems were studied in [7] with the use of the fermi-liquid approach proposed by Landau.

\section{Spatially periodical Bose condensation in the model of a weakly nonideal Bose gas}

In this section we consider the generalization of the Bogolyubov theory of a weakly nonideal Bose gas for the case when the Bose condensate consists of particles with the infinite set of momenta $\vec{p}+\vec{\tau}$, where vectors $\vec{\tau}$ form a certain reciprocal lattice with respect to a direct one with vectors $\vec{a}_{i}, i=1,2,3\left(\vec{\tau}=\sum_{i=1}^{3} n_{i} \vec{b}^{i}, n_{i}\right.$ are the integer numbers, $\vec{b}^{i} \vec{a}_{j}=2 \pi \delta_{i j}$ ) and the vector $\vec{p}$ belongs to the first Brillouin zone. To study this problem we use the quasiaverage method leading to the model with an isolated condensate.

According to this method, an average value of arbitrary function $\hat{A}(\hat{\psi})$ of the annihilation operator $\hat{\psi}(\vec{x})$ can be defined by the formula

$$
\{\hat{A}(\hat{\psi})\}=\operatorname{Sp} \hat{w}(\psi) \hat{A}(\psi)
$$

where the operator $\hat{A}(\psi)$ can be obtained from the operator $\hat{A}(\hat{\psi})$ by the substitution

$$
\hat{\psi}(\vec{x}) \rightarrow \psi(\vec{x})+\hat{\psi}^{\prime}(\vec{x})
$$

where

$$
\hat{\psi}^{\prime}(\vec{x})=\frac{1}{\sqrt{V}} \sum_{\vec{q} \neq \vec{p}+\vec{\tau}} \hat{a}_{\vec{q}} \mathrm{e}^{\mathrm{i} \vec{q} \vec{x}}
$$

and the quantity $\psi(\vec{x})$ represents a C-numerical function

$$
\psi(\vec{x})=\frac{1}{\sqrt{V}} \sum_{\vec{q}=\vec{p}+\vec{\tau}} a_{\vec{q}} \mathrm{e}^{\mathrm{i} \vec{q} \vec{x}}, \quad\left(a_{\vec{q}} \sim \sqrt{V}\right)
$$

and

$$
\begin{gathered}
\hat{w}(\psi)=\exp (\Omega(\psi)-\beta(\hat{H}(\psi)-\vec{v} \hat{\vec{P}}(\psi)-\mu \hat{N}(\psi))) \\
\Omega(\psi)=-\ln \operatorname{Sp} \exp \beta(\hat{H}(\psi)-\vec{v} \overrightarrow{\vec{P}}(\psi)-\mu \hat{N}(\psi)) .
\end{gathered}
$$


Here $\hat{H}(\psi)$ is the Hamilton operator, $\hat{\vec{P}}(\psi)$ is the operator of momenta, and $\hat{N}(\psi)$ is the operator of number of particles. The function $\psi(\vec{x})$ (or quantity $a_{\vec{p}+\vec{\tau}}$ ) can be determined from a minimum condition of the potential $\Omega(\psi)$ :

$$
\frac{\delta \Omega}{\delta \psi}=0, \quad \frac{\delta \Omega}{\delta \psi^{*}}=0
$$

In the weakly nonideal gas approximation we can write

$$
\begin{aligned}
\hat{H}(\psi) \approx H(\psi)= & \frac{1}{2 m} \int \mathrm{d} \vec{x} \nabla \psi^{*}(\vec{x}) \nabla \psi(\vec{x}) \\
& +\frac{1}{2} \int \mathrm{d} \vec{x}_{1} \int \mathrm{d} \vec{x}_{2} \psi^{*}\left(\vec{x}_{1}\right) \psi^{*}\left(\vec{x}_{2}\right) \nu\left(\vec{x}_{1}-\vec{x}_{2}\right) \psi\left(\vec{x}_{1}\right) \psi\left(\vec{x}_{2}\right), \\
\hat{\vec{P}}(\psi) \approx \vec{P}(\psi)= & -\frac{\mathrm{i}}{2} \int \mathrm{d} \vec{x}\left(\psi^{*}(\vec{x}) \nabla \psi(\vec{x})-\nabla \psi^{*}(\vec{x}) \psi(\vec{x})\right), \\
\hat{N}(\psi) \approx N(\psi)= & \int \mathrm{d} \vec{x} \psi^{*}(\vec{x}) \psi(\vec{x}) .
\end{aligned}
$$

Then, according to (2.6) the equation for determining the quantity $\psi(\vec{x})$ has the form

$$
\left\{-\frac{1}{2 m} \Delta+\mathrm{i} \vec{v} \nabla-\mu+\nu|\psi(\vec{x})|^{2}\right\} \psi(\vec{x})=0
$$

(we suppose that the function $\psi(x)$ slightly changes in the course of the interaction radius $\left.r_{0}\right)$. If we introduce the function $u(\vec{x})$ instead of $\psi(\vec{x})$

$$
\psi(\vec{x})=\mathrm{e}^{\mathrm{i} \vec{p} \vec{x}} u(\vec{x})
$$

where $u(\vec{x})$ is the periodical function of $\vec{x}$

$$
u(\vec{x}+\vec{a})=u(\vec{x}),
$$

which can be represented in the form

$$
u(\vec{x})=\eta(\vec{x}) \mathrm{e}^{\mathrm{i} \varphi(\vec{x})},
$$

then we obtain the following equations for determining the functions $\eta, \varphi$ :

$$
\begin{aligned}
-\frac{1}{2 m} \Delta \eta+\frac{\eta}{2 m}(\nabla \varphi)^{2}-\eta \widetilde{\vec{v}} \nabla \varphi-\widetilde{\mu} \eta+\nu \eta^{3} & =0, \\
-\frac{1}{m} \nabla \eta \nabla \varphi-\frac{\eta}{2 m} \Delta \varphi+\widetilde{\vec{v}} \nabla \eta & =0,
\end{aligned}
$$

where

$$
\widetilde{\vec{v}}=\vec{v}-\frac{\vec{p}}{m}, \quad \widetilde{\mu}=\mu+\vec{p} \vec{v}-\frac{\vec{p}^{2}}{2 m} .
$$

Further, it will be supposed that the condensate has got only one period $X$ along the axis $x$ and the system is spatially homogeneous with respect to the axes $y$ and $z$. 
Bearing in mind this supposition, the solution of the last equation from (2.12) can be written as

$$
\varphi(x)=m \widetilde{v}_{\|} x+C \int_{a}^{x} \frac{\mathrm{d} x}{\zeta},
$$

where $\zeta(x)=\eta^{2}(x), \widetilde{v}_{\|}=\widetilde{v}_{x}$. The integration constant $C$, equalled to

$$
C=(2 \pi n-m \widetilde{v} X) / \int_{0}^{X} \frac{\mathrm{d} x}{\zeta}
$$

is defined from the "periodical" condition of the function $\varphi(x)$

$$
\varphi(x+X)=\varphi(x)+2 \pi n,
$$

where $n$ is an arbitrary integer and a fixed number. This number determines different solutions (or phases) of equations (2.12). Substituting the function $\varphi(x)$ into the first equation from (2.12) we get:

$$
-\frac{1}{2 m} \frac{\mathrm{d}^{2} \eta}{\mathrm{d} x^{2}}+\frac{C^{2}}{2 m} \frac{1}{\eta^{3}}-\eta \mu^{*}+\nu \eta^{3}=0,
$$

where

$$
\mu^{*}=\mu+\frac{m v^{2}}{2}-\frac{m \widetilde{\vec{v}}_{\perp}^{2}}{2}, \quad \widetilde{\vec{v}}_{\perp}=\widetilde{\vec{v}}-\widetilde{\vec{v}}_{\|} .
$$

Multiplying (2.16) by $\mathrm{d} \eta / \mathrm{d} x$, we find the first integral:

$$
\left(\frac{\mathrm{d} \zeta}{\mathrm{d} x}\right)^{2}=f(\zeta)
$$

where

$$
\begin{aligned}
f(\zeta) & =4 \mu \nu\left(\zeta^{3}-2 \frac{\mu^{*}}{\nu} \zeta^{2}+2 \frac{\mathcal{E}}{\nu} \zeta-\frac{C^{2}}{m \nu}\right) \\
& \equiv 4 \mu \nu\left(\zeta-\zeta_{1}\right)\left(\zeta-\zeta_{2}\right)\left(\zeta-\zeta_{3}\right),
\end{aligned}
$$

moreover

$$
\zeta_{1}+\zeta_{2}+\zeta_{3}=\frac{2 \mu^{*}}{\nu}, \quad \zeta_{1} \zeta_{2}+\zeta_{1} \zeta_{3}+\zeta_{2} \zeta_{3}=\frac{2 \mathcal{E}}{\nu}, \quad \zeta_{1} \zeta_{2} \zeta_{3}=\frac{C^{2}}{m \nu}
$$

( $\mathcal{E}$ is the integration constant).

Next, it will be shown that for achieving stability of the periodical Bose condensate with respect to a normal state, the following conditions

$$
\nu>0, \quad \mu^{*}>0
$$

should be fulfilled (see (3.4)). The first inequality leads to

$$
f(0)<0
$$


It is necessary to have all the three roots $\zeta_{i}$ real and positive for the existence of the periodical solutions $\zeta(x)>0$ of equation (2.18). Moreover, the positive roots $\zeta_{i}$ can be always chosen so that the following inequalities will be valid

$$
0<\zeta_{1}<\zeta_{2}<\zeta_{3} \text {. }
$$

Let us also note that the inequality $\mathcal{E}>0$ holds true. It is evident that the periodical solution $\zeta(x)$ of equation (2.18) lies between $\zeta_{1}$ and $\zeta_{2}$

$$
\zeta_{1}<\zeta(x)<\zeta_{2}
$$

Solution of equation (2.18) for $\zeta(x)$ in terms of Jacobi's elliptic functions has the form

$$
\zeta(x)=\zeta_{1}+\left(\zeta_{2}-\zeta_{1}\right) \operatorname{sn}^{2}(u, k),
$$

where

$$
u=\frac{2 K(k)}{X} x, \quad k^{2}=\frac{\zeta_{2}-\zeta_{1}}{\zeta_{3}-\zeta_{1}}
$$

and $K(k)$ is the full elliptic integral of the first type

$$
K(k)=\int_{0}^{\pi / 2} \frac{\mathrm{d} \varphi}{\sqrt{1-k^{2} \sin ^{2} \varphi}} .
$$

We want to emphasize that the function $\zeta(x)$ has a period which equals to $X$ due to the formula

$$
\operatorname{sn}(u+2 K(k), k)=-\operatorname{sn}(u, k) .
$$

Let us turn to determining the phase $\varphi(x)$. According to (2.14) we have

$$
\varphi(x)=m \widetilde{v}_{\|} x+\left(2 \pi n-m \widetilde{v}_{\|} X\right) Z(x),
$$

where

$$
Z(x)=\int_{0}^{x} \frac{\mathrm{d} x}{\zeta} / \int_{0}^{X} \frac{\mathrm{d} x}{\zeta} .
$$

Proceeding from the last formula for the function $Z(x)$ it is easy to conclude that

$$
Z(x)=l+Z_{0}(x-l X), \quad l X<x<(l+1) X .
$$

Here

where

$$
Z_{0}(x)= \begin{cases}\frac{1}{2} Y(x), & 0<x<\frac{X}{2} \\ 1-\frac{1}{2} Y(x), & \frac{X}{2}<x<X\end{cases}
$$

$$
\begin{aligned}
Y(x)=\left(\int_{\zeta_{1}}^{\zeta_{2}} \frac{\mathrm{d} \zeta}{\zeta \sqrt{f}}\right)^{-1} \int_{\zeta_{1}}^{\zeta(x)} \frac{\mathrm{d} \zeta}{\zeta \sqrt{f}}=\frac{G\left(\theta(x), \frac{\zeta_{2}}{\zeta_{1}}-1, k\right)}{G\left(\frac{\pi}{2}, \frac{\zeta_{2}}{\zeta_{1}}-1, k\right)}, \\
\theta(x) \equiv \theta(\zeta(x))=\arcsin \sqrt{\frac{\zeta(x)-\zeta_{1}}{\zeta_{2}-\zeta_{1}}}
\end{aligned}
$$

and $G(\theta, h, k)$ is the elliptic integral of the third type

$$
G(\theta, h, k)=\int_{0}^{x} \frac{\mathrm{d} x}{\left(1+h \sin ^{2} x\right) \sqrt{1-k^{2} \sin ^{2} x}} .
$$

Expressions (2.24)-(2.27) along with formula (2.11) determine the structure of the function $u(x)$. 


\section{Thermodynamics of the spatially periodical Bose condensate}

According to (2.5), the density of the Gibbs thermodynamic potential $\omega$ in a model with the isolated condensate is defined by the formula

$$
\omega=\frac{\Omega}{V \beta}=-\frac{1}{V \beta} \ln \operatorname{Sp} \exp [-\beta(\hat{H}(\psi)-\vec{v} \hat{\vec{P}}(\psi)-\mu \hat{N}(\psi))]
$$

or in the approximation of a weakly nonideal Bose gas we can write

$$
\omega=\frac{1}{V}(H(\psi)-\vec{v} \vec{P}(\psi)-\mu N(\psi))
$$

where $H(\psi), \vec{P}(\psi), N(\psi)$ are defined by (2.7). Therefore, using (2.24)-(2.27), (2.11) we find

$$
\omega=\frac{1}{X} \int_{0}^{X} \mathrm{~d} x\left\{-\frac{\eta}{2 m} \frac{\mathrm{d}^{2} \eta}{\mathrm{d} x^{2}}+\frac{1}{2 m} \frac{C^{2}}{\eta^{2}}+\frac{1}{2} \nu \eta^{4}-\mu^{*} \eta^{2}\right\}, \quad \mu^{*}=\mu+\frac{m \vec{v}^{2}}{2}-\frac{m \widetilde{\vec{v}}_{\perp}^{2}}{2} .
$$

Excluding $\mathrm{d}^{2} \eta / \mathrm{d} x^{2}$ with the help of (2.16) we get

$$
\omega=-\frac{\nu}{2 X} \int_{0}^{X} \mathrm{~d} x \eta^{4}=-\frac{\nu}{2 X} \int_{0}^{X} \mathrm{~d} x \zeta^{2} .
$$

Since the potential of a normal state is equal to zero (see formula (3.2), where $\psi=0$ ), the condition of the thermodynamic stability of the considered spatially-periodical Bose condensate has the form

$$
\omega<0
$$

Therefore, from (2.24) it follows that $\nu>0$, and from (2.23) that $\mu^{*}>0$.

Let us obtain an explicit form for the thermodynamic potential $\omega$. Changing from the integration variable $x$ to $\zeta$ in the integrand of formula (3.4) and using (2.18), (2.19) we find

$$
\omega=-\frac{\nu}{X} \int_{\zeta_{1}}^{\zeta_{2}} \mathrm{~d} \zeta \frac{\mathrm{d} x}{\mathrm{~d} \zeta} \zeta^{2}=-\frac{\nu}{X} \int_{\zeta_{1}}^{\zeta_{2}} \mathrm{~d} \zeta \frac{\zeta^{2}}{\sqrt{f(\zeta)}}
$$

Calculating the integral entering this formula, it can be shown that

$$
\begin{gathered}
\omega(\zeta)=\frac{\nu}{6}\left\{\zeta_{1} \zeta_{2}+\zeta_{1} \zeta_{3}+\zeta_{2} \zeta_{3}-2 \zeta_{3}\left(\zeta_{1}+\zeta_{2}+\zeta_{3}\right)+2\left(\zeta_{3}-\zeta_{1}\right)\left(\zeta_{1}+\zeta_{2}+\zeta_{3}\right) \frac{E(k)}{K(k)}\right\} \\
k^{2}=\frac{\zeta_{2}-\zeta_{1}}{\zeta_{3}-\zeta_{1}}
\end{gathered}
$$

where $E(k)$ is the full elliptic integral of the second type.

$$
E(k)=\int_{0}^{\pi / 2} \mathrm{~d} \varphi \sqrt{1-k^{2} \sin ^{2} \varphi}
$$


Density of the condensate particles is given by the formula

$$
\frac{1}{X} \int_{0}^{X} \mathrm{~d} x|\psi(x)|^{2}=\frac{1}{V} \sum_{\vec{\tau}}\left|a_{\vec{p}+\vec{\tau}}\right|^{2} \equiv n_{0}=\frac{1}{X} \int_{0}^{X} \mathrm{~d} x \zeta(x)=\frac{2}{X} \int_{\zeta_{1}}^{\zeta_{2}} \mathrm{~d} \zeta \frac{\zeta}{\sqrt{f(\zeta)}}
$$

or

$$
n_{0}=\zeta_{3}-\left(\zeta_{3}-\zeta_{1}\right) \frac{E(k)}{K(k)}
$$

Thus, we can use the variables $\zeta_{1}, \zeta_{2}, \zeta_{3}$ instead of the thermodynamic variables $\mu^{*}, \widetilde{\vec{v}}_{\|}, X$. The obtained quantities $\omega, n_{0}$ are the functions of these new variables $\zeta_{1}, \zeta_{2}, \zeta_{3}$. It can be easily seen that the variables $\zeta_{1}, \zeta_{2}, \zeta_{3}$ and $\mu^{*}, \widetilde{v}_{\|}, X$ related by

$$
\begin{gathered}
\frac{K(k)}{\sqrt{\zeta_{3}-\zeta_{1}}}=\frac{1}{2} \sqrt{m \nu} X, \quad \zeta_{1}+\zeta_{2}+\zeta_{3}=\frac{2 \mu^{*}}{\nu}, \\
\frac{2}{\zeta_{1} \sqrt{\zeta_{3}-\zeta_{1}}} \sqrt{\zeta_{1} \zeta_{2} \zeta_{3}} G\left(\frac{\zeta_{2}}{\zeta_{1}}-1, k\right)=\left|2 \pi n-m \widetilde{v}_{||} X\right|,
\end{gathered}
$$

where $G(h, k)$ is the full elliptic integral of the third type

$$
G(h, k)=\int_{0}^{\pi / 2} \frac{\mathrm{d} \varphi}{\left(1+h \sin ^{2} \varphi\right) \sqrt{1-k^{2} \sin ^{2} \varphi}} .
$$

Using expression (3.6) for the thermodynamic potential $\omega$ one can obtain a thermodynamic identity

$$
\delta \omega=-\frac{1}{X}\left(\left\langle\frac{1}{m}\left(\frac{\partial \eta}{\partial x}\right)^{2}\right\rangle+\frac{2 \pi n C}{m X}\right) \delta X-C \delta \widetilde{v}_{\|}-n_{0} \delta \mu^{*}
$$

which represents the second thermodynamic law for reversible processes.

We also want to write a following formula for the average value of the density of momenta

$$
\vec{\pi}=-\frac{\mathrm{i}}{2 X} \int_{0}^{X} \mathrm{~d} x\left\{\psi^{*} \nabla \psi-\nabla \psi^{*} \psi\right\}
$$

It yields

$$
\pi_{\|}=n_{0} m v_{\|}+C, \quad \vec{\pi}_{\perp}=n_{0} \vec{v}_{\perp} .
$$

Thus, a physical meaning of the constant $C$ (see (2.15)) determining the component of momenta along the $x$ axis is shown. As we can see, the transverse component of momenta $\vec{\pi}_{\perp}$ coincides with the density of momenta for a superfluid movement (we take into account that the density of the normal component is equal to zero in the approximation considered).

The above mentioned formulae can be simplified for the limit $\zeta_{1} \rightarrow 0$. In this case according to $(2.24) k^{2}=\zeta_{2} / \zeta_{3}$ and according to $(2.23)$ the function $\zeta(x)$ has the form:

$$
\zeta(x)=\zeta_{2} \operatorname{sn}^{2}\left(\frac{x}{X} 2 K(k)\right)
$$


and the phase $\varphi(x)$ is given by

$$
\varphi(x)=m \widetilde{v}_{\|} x+\left(2 \pi n-m \widetilde{v}_{\|} X\right)\left(l+\frac{1}{2}\right), \quad l X<x<(l+1) X .
$$

Bearing in mind (2.20) we have

$$
\zeta_{2}+\zeta_{3}=\frac{2 \mu^{*}}{\nu}, \quad \zeta_{2} \zeta_{3}=\frac{2 \mathcal{E}}{\nu}
$$

wherefrom one can find

$$
\zeta_{2}=\frac{\mu^{*}}{\nu}\left(1-\sqrt{1-\frac{\mathcal{E}}{U_{0}}}\right), \quad \zeta_{3}=\frac{\mu^{*}}{\nu}\left(1+\sqrt{1-\frac{\mathcal{E}}{U_{0}}}\right),
$$

where $U_{0}=\mu^{* 2} / 2 \nu$. These formulae lead to

$$
k^{2}=\frac{\zeta_{2}}{\zeta_{3}}=\frac{1-\sqrt{1-\frac{\mathcal{E}}{U_{0}}}}{1+\sqrt{1-\frac{\mathcal{E}}{U_{0}}}}
$$

and, consequently,

$$
\zeta_{2}=\frac{\mu^{*}}{\nu} \frac{2 k^{2}}{1+k^{2}}, \quad \zeta_{3}=\frac{\mu^{*}}{\nu} \frac{2}{1+k^{2}} .
$$

Therefore, formulae (3.7), (3.12) imply that

$$
\omega\left(\mu^{*}, k\right)=\frac{2}{3} \frac{\mu^{* 2}}{\nu} \frac{1}{1+k^{2}}\left(2 \frac{E(k)}{K(k)}-\frac{2+k^{2}}{1+k^{2}}\right), \quad X=\frac{2}{\sqrt{2 m \mu^{*}}} \sqrt{1+k^{2}} K(k) .
$$

The quantity $\widetilde{v}_{\|}$entering $\varphi(x)$ should be associated with the infinitesimal quantity $\zeta_{1}$. In order to set this connection let us return to formula (3.10) and find the asymptotes of the function $G\left(\zeta_{2} / \zeta_{1}-1, k\right)$ at $\zeta_{1} \rightarrow 0$. To this end we note that by means of an operation of integration by part it is easy to prove the validity of the relation

$$
\begin{aligned}
\int_{0}^{\pi / 2} & \frac{\mathrm{d} \varphi}{\left(\zeta_{1}+\chi_{2} \sin ^{2} \varphi\right) \sqrt{1-k^{2} \sin ^{2} \varphi}}= \\
& =\int_{0}^{\pi / 2} \frac{\mathrm{d} \varphi}{\zeta_{1}+\chi_{2} \sin ^{2} \varphi}+\int_{0}^{\pi / 2} \mathrm{~d} \varphi \frac{k^{2} \sin \varphi \cos \varphi}{\left(1-k^{2} \sin ^{2} \varphi\right)^{3 / 2}} \int_{\varphi}^{\pi / 2} \frac{\mathrm{d} \varphi}{\zeta_{1}+\chi_{2} \sin ^{2} \varphi}
\end{aligned}
$$

here $\left(\chi_{2}=\zeta_{2}-\zeta_{1}\right)$. Using this formula we have

$$
\zeta_{1}^{-1} G\left(\frac{\zeta_{2}}{\zeta_{1}}-1 ; k\right) \underset{\zeta_{1} \rightarrow 0}{\longrightarrow} \frac{\pi}{2} \frac{1}{\sqrt{\zeta_{1}}}+\frac{K(k)-E(k)}{\zeta_{2}} .
$$

This yields

$$
\frac{2 \sqrt{\zeta_{1} \zeta_{2} \zeta_{3}}}{\zeta_{1} \sqrt{\zeta_{3}-\zeta_{1}}} G\left(\frac{\zeta_{2}}{\zeta_{1}}-1 ; k\right) \underset{\zeta_{1} \rightarrow 0}{\longrightarrow} \pi+2 \frac{K(k)-E(k)}{\sqrt{\zeta_{2}}} \sqrt{\zeta_{1}}
$$


Thus, formula (3.10) takes the form

$$
2 \frac{K(k)-E(k)}{\sqrt{\zeta_{2}}} \sqrt{\zeta_{1}}=2 \pi(n-1 / 2)-m \widetilde{v}_{\|} X>0
$$

(the case $2 \pi(n-1 / 2)-m \widetilde{v}_{\|} X<0$ is not realized). In such a way $m \widetilde{v}_{\|} X \rightarrow 2 \pi(n-1 / 2)$ at $\zeta_{1} \rightarrow 0$ and consequently (see $(2.27)$ )

$$
\varphi(x)=m \widetilde{v} x+\pi Z_{0}(x)=2 \pi(n-1 / 2) \frac{x}{X}+\pi\left(l+\frac{1}{2}\right), \quad l X<x<(l+1) X .
$$

Let us find now the function $u(x) \equiv \mathrm{e}^{\mathrm{i} \varphi(x)} \sqrt{\zeta(x)}$. According to $(2.11),(2.23)$ we can write

$$
u(x)=\sqrt{\zeta_{2}}\left|\operatorname{sn}\left(\frac{x}{X} K(k)\right)\right| \mathrm{e}^{\mathrm{i} \varphi(x)}=\mathrm{i} \sqrt{\zeta_{2}} \operatorname{sn}\left(\frac{x}{X} 2 K(k)\right) \exp (-\mathrm{i} \pi x / X) \exp (2 \pi n \mathrm{i} x / X),
$$

where $\operatorname{sn}(x 2 K(k) / X) \exp (-\mathrm{i} \pi x / X)$ is the periodical function of $x$ with the period $X$ due to $\operatorname{sn}(u+2 K(k))=-\operatorname{sn} u$. The period $X$ is given by the formula $X=\left(2 / \sqrt{2 m \mu^{*}}\right)$ $\times \sqrt{1+k^{2}} K(k)$. Expanding the periodical function $\operatorname{sn}(x 2 K(k) / X) \exp (-\mathrm{i} \pi x / X)$ into the Fourier series

$$
\exp \left(-\mathrm{i} \pi \frac{x}{X}\right) \operatorname{sn}\left(\frac{2 x}{X} K(k)\right)=\sum_{m} b_{m} \exp \left(2 \pi \mathrm{i} m \frac{x}{X}\right),
$$

where

$$
\begin{gathered}
2 \pi b_{m}=\int_{-\pi}^{\pi} \mathrm{d} t \mathrm{e}^{-\mathrm{i}(m+1 / 2) t} \mathrm{sn}\left(\frac{t}{\pi} K(k)\right)=-\frac{\mathrm{i} \pi^{2}}{k K}\left(\operatorname{sh}\left(n+\frac{1}{2}\right) \pi \frac{K^{\prime}}{K}\right)^{-1}, \\
K^{\prime}=\left.K\right|_{k^{2} \rightarrow 1-k^{2}}
\end{gathered}
$$

we obtain

$$
u(x)=\mathrm{i} \sqrt{\zeta_{2}} \exp \left(2 \pi \mathrm{i} n \frac{x}{X}\right) \sum_{m} b_{m} \exp \left(2 \pi \mathrm{i} m \frac{x}{X}\right) .
$$

The last formula with (2.4) indicate that a population of the states by condensate particles with the momenta $\vec{p}+\vec{i} 2 \pi(m+n) / X$ is determined by the formula

$$
\left|a_{\vec{p}+\vec{i} 2 \pi m / X}\right|^{2}=V \frac{2 \mu^{*}}{\nu} k^{2}\left|b_{m}\right|^{2} .
$$

Now let us study the problem concerning the stability of the Bose condensate. As it was mentioned the inequality

$$
\omega(\zeta)<0
$$

takes place at $\nu>0$. Since the potential $\omega=0$ corresponds to a normal state (the order parameter $\psi$ turns to zero for the normal state), the spatially periodical Bose condensate is thermodynamically stable with respect to the normal state. 
Let us consider the phase with $n=0$. Then according to (3.11)

$$
\frac{\partial \omega}{\partial X}=-\frac{1}{m X}\left\langle\left(\frac{\partial \eta}{\partial x}\right)^{2}\right\rangle<0
$$

It means that $\omega$ is the decrease function of the period $X$. Thus, at $n=0$ the spatially homogeneous state with $X=\infty$ is an absolutely stable state. This state corresponds to the Bogolyubov solution. However, the mentioned fact does not mean that the spatially periodical solutions do not realize even in the case $n=0$. Really, the considered spatially periodical solutions correspond to the statistical operator $\hat{\rho}$ which is invariant with respect to the unitary transformation $U=\exp i X\left(\hat{P}_{x}-p_{x} \hat{N}\right)$

$$
U \rho U^{+}=\rho
$$

and, consequently, in virtue of the conservation laws for $\hat{P}_{x}$ and $\hat{N}$, the period $X$ is the motion constant and cannot change if the conservation law for $\hat{P}_{x}$ is not violated. But if the conservation law for $\hat{P}_{x}$ is weakly violated, then the studied spatially periodical states become quasistationary ones and parameter $X$ will be slowly turned to infinity (in virtue of the weakly violation of the conservation law for $\hat{P}_{x}$ ). That is why the considered spatially periodical solution turns to the Bogolyubov spatially homogeneous solution at $t \rightarrow \infty$.

This is similar to the situation for the normal state when the term $-\vec{v} \hat{\vec{P}}$ is added to the Gibbs exponent. The quantity $\vec{v}$ is the motion constant and the state is stable until the conservation law is violated. But the state becomes quasistationary and parameter $\vec{v}$ turns to zero at the weakly violation of the momenta conservation law.

Even a more complicated situation can arise for phases with $n \neq 0$. In this case the condition $\partial \omega / \partial X<0$ becomes not evident and, hence, the possibility of the existence of absolutely stable and spatially periodical solutions with a definite value of the period $X$ can appear. The period $X$ is not an independent thermodynamic parameter (as in quantum crystals) anymore, but it can be treated as a certain function of $\mu^{*}, \widetilde{v} ; X=X\left(\mu^{*}, \widetilde{v}_{\|}\right)$.

\section{Acknowledgements}

This work was supported in part by the German Ministry of Science and Technology (BMBF: 4114006 06ROS02), and also by the Ukrainian State Foundation for Fundamental Studies.

\section{References}

1. Anderson M.H., Ensher I.R., Mattews M.R. et al. Observation of Bose-Einstein condensation in a dilute atomic vapors. // Science, 1995, vol. 269, p. 198-201.

2. Bogolyubov N.N. On the theory of superfluidity. // Izv. Akad. Nauk. SSSR, ser. fiz., 1947, vol. 11, No. 1, p. 77-90 (in Russian).

3. Bogolyubov N.N. Quasiaverages in the problems of statistical mechanics. Preprint of Joint Institute of Nuclear Researches, JINR-1451, Dubna, 1963, 123 p. (in Russian). 
4. Lavrinenko N.M., Peletminsky S.V. Thermodynamics and motion equations of quantum crystals. // Teor. Mat. Fiz., 1986, vol. 66, No. 2, p. 314-325 (in Russian).

5. Andreev A.F., Lifshitz I.M. Quantum theory of crystal defects. // Zh. Eksp. Teor. Fiz., 1969, vol. 56, No. 6, p. 2057-2068 (in Russian).

6. Akhiezer A.I., Peletminskii S.V., Slyusarenko Yu.V. Theory of a weakly nonideal Bose gas in a magnetic field. // JETP, 1998, vol. 86, No. 3, p. 501-506.

7. Peletminsky A.S., Peletminsky S.V., Slyusarenko Yu.V. On phase transitions in a Fermi liquid. II. Transition associated with translational symmetry breaking. // Low Temperature Physics, 1999, vol. 25, No. 5, p. 303-313.

\title{
Про можливІсть Існування просторово перІодичного бозе-конденсату
}

\author{
О.С.Пелетминський ${ }^{1}$, С.В.Пелетминський ${ }^{2}$, \\ Ю.В.Слюсаренко ${ }^{2}$
}

1 Науково-технічний центр електрофізичної обробки НАН України, 61002 Харків

2 Національний науковий центр

"Харківський фізико-технічний інститут",

61108 Харків

Отримано 6 березня 2000 р.

У роботі вивчено можливість побудови теорії просторово-періодичного бозе-конденсату в моделі слабконеідеального бозе-газу. На основі методу виділеного бозе-конденсату сформульовано рівняння для визначення просторово-періодичного параметра порядку при температурі $T=0$, коли можна знехтувати внеском квазічастинок до термодинаміки системи бозонів, та знайдено їх одноперіодичні розв'язки. Коротко викладено питання термодинамічної стійкості просторово-періодичного бозе-конденсату.

Ключові слова: слабко неідеальний бозе-газ, метод виділеного бозе-конденсату, просторово періодичний бозе-конденсат, термодинамічна стійкість

PACS: 03.75.Fi, 05.30.Jp, 05.70.a 
